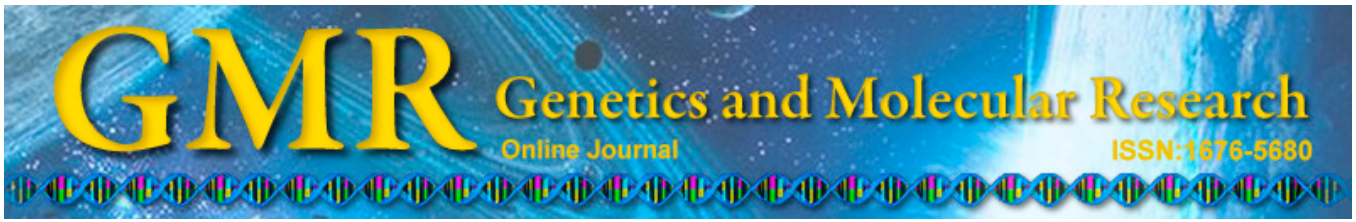

\title{
Meta-analysis of differentially expressed genes in ankylosing spondylitis
}

\author{
Y.H. Lee and G.G. Song \\ Division of Rheumatology, Department of Internal Medicine, \\ Korea University College of Medicine, Seoul, Korea \\ Corresponding author: Y.H. Lee \\ E-mail: lyhcgh@korea.ac.kr
}

Genet. Mol. Res. 14 (2): 5161-5170 (2015)

Received August 11, 2014

Accepted January 19, 2015

Published May 18, 2015

DOI http://dx.doi.org/10.4238/2015.May.18.6

\begin{abstract}
The purpose of this study was to identify differentially expressed (DE) genes and biological processes associated with changes in gene expression in ankylosing spondylitis (AS). We performed a metaanalysis using the integrative meta-analysis of expression data program on publicly available microarray AS Gene Expression Omnibus (GEO) datasets. We performed Gene Ontology (GO) enrichment analyses and pathway analysis using the Kyoto Encyclopedia of Genes and Genomes. Four GEO datasets, including 31 patients with AS and 39 controls, were available for the meta-analysis. We identified 65 genes across the studies that were consistently DE in patients with AS vs controls (23 upregulated and 42 downregulated). The upregulated gene with the largest effect size (ES; -1.2628, $\mathrm{P}=0.020951)$ was integral membrane protein $2 \mathrm{~A}(I T M 2 A)$, which is expressed by CD4+ $\mathrm{T}$ cells and plays a role in activation of $\mathrm{T}$ cells. The downregulated gene with the largest ES (1.2299, $\mathrm{P}=0.040075)$ was mitochondrial ribosomal protein S11 (MRPS11). The most significant GO enrichment was in the respiratory electron transport chain category $\left(\mathrm{P}=1.67 \times 10^{-9}\right)$. Therefore, our metaanalysis identified genes that were consistently DE as well as biological pathways associated with gene expression changes in AS.
\end{abstract}

Key words: Ankylosing spondylitis; Gene expression; Meta-analysis; Pathway analysis 


\section{INTRODUCTION}

Ankylosing spondylitis (AS) is a chronic inflammatory disorder characterized by inflammation in the spine and sacroiliac joints resulting in initial bone and joint erosion and subsequent ankylosis (Brown et al., 2002). Human leukocyte antigen (HLA) B27 was the first genetic factor to be associated with AS, and it confers considerable susceptibility to the disease. However, there has been increasing evidence to suggest that non-HLA-B27 genes also contribute to AS pathogenesis (Lee et al., 2005a). Strong genetic factors have been implicated in the etiology of AS, but the biological mechanisms associated with the disease are still unclear.

High-throughput genomics technologies such as microarrays have improved our understanding of complex gene interactions and networks during disease development. Microarrays measure the expression of thousands of genes simultaneously on a genome-wide scale (Golub et al., 1999). Alterations in genetic profiles can be correlated to altered gene functions and biochemical activities. Microarray technology is a powerful tool that has become one of the most frequently used investigational methods in medical research.

Identification of gene expression signatures that differentiate disease states from those of healthy controls are dependent on sample availability, sample size, and heterogeneous datasets (Ramasamy et al., 2008). Although many microarray studies have produced lists of differentially expressed (DE) genes, there tend to be inconsistencies between studies due to the limitations of small sample sizes and variable results (Siddiqui et al., 2006).

To address these challenges, meta-analysis can be performed using publically available data from genome-wide gene expression studies in specific diseases (Griffith et al., 2006; Rung and Brazma, 2013). Meta-analysis can enhance the reliability and generalizability of studies to obtain a more precise estimate of gene expression profiles (Griffith et al., 2006). Meta-analysis enhances the statistical power for identifying more robust and reliable gene signatures (Lee and Nath, 2005; Lee et al., 2005b; Choi et al., 2006). Recently, a new user-friendly microarray meta-analysis tool called integrative meta-analysis of expression data (INMEX) has been developed to support the meta-analysis of multiple gene expression datasets, as well as to enable the integration of datasets of gene expression and pathways (Xia et al., 2013).

In order to overcome the limitations of individual studies, resolve inconsistencies, and reduce the likelihood of false-positive or -negative associations caused by random errors, we performed a microarray meta-analysis with the objective to identify DE genes in AS and biological processes associated with these gene expression changes.

\section{MATERIAL AND METHODS}

\section{Identification of eligible AS gene expression datasets}

A search for microarray datasets that examined DE genes between patients with AS and controls was conducted. We utilized the NCBI Gene Expression Omnibus (GEO) database (http://www.ncbi.nlm.nih.gov/geo/) to identify microarray datasets submitted through September, 2013 (Barrett et al., 2011). The key word "ankylosing spondylitis" was used in the search. Studies were included in the analysis if they met the following criteria: 1) case-control studies; 2) included gene expression data; and 3) included patients diagnosed with AS based on specific AS diagnostic criteria (van der Linden et al., 1984). We excluded animal studies and studies in which microarray data could not be obtained. The following information was 
extracted from each identified study: GEO accession number, sample type, platform, number of cases and controls, references, and gene expression data.

\section{Meta-analysis of microarray datasets}

All available AS microarray datasets were downloaded from the NCBI GEO database. We constructed data tables containing gene expression values or relative expression values with genes/probes in rows and samples/experiments delineated in individual columns. After uploading the datasets into the INMEX program (http://inmex.ca/INMEX) (Xia et al., 2013), we annotated the data by converting different gene or probe IDs to Entrez IDs. For each probe set, intensity values were log-transformed and normalized to zero mean and unit variance (Bolstad et al., 2003). When all datasets were uploaded, processed, and annotated, we performed a data integrity check before proceeding to the meta-analysis stage. The random-effects model, which assumes that individual studies contain substantial diversity and assesses both withinstudy sampling error and between-study variance (DerSimonian and Laird, 1986; Choi et al., 2003), is used in the presence of significant between-study heterogeneity (DerSimonian and Laird, 1986). Statistical manipulations were undertaken using INMEX.

\section{Functional analysis}

To further understand the functions of the genes present in the data lists, we performed GO enrichment analysis using hypergeometric tests (http://www.geneontology.org/) (Falcon and Gentleman, 2007). To functionally annotate the genes in the list, we identified over-represented Kyoto Encyclopedia of Genes and Genomes (KEGG) categories (http://www.genome. ad.jp/) (Hua et al., 2010). Functional analysis was undertaken using INMEX.

\section{RESULTS}

\section{Studies included in the meta-analysis}

Eighty-five studies were identified by electronic search, eight of which were selected for full dataset review based on title and abstract details. Four datasets were excluded because they contained no data for meta-analysis, and four microarray datasets met our inclusion criteria (Table 1). These datasets consisted of results from two synovium and two blood sample studies, and included a total of 31 patients with AS and 39 controls. Selected details of the individual studies are summarized in Table 1. Heat maps using differential expression analysis of individual datasets were used to visualize a subset of genes across the four studies (Figure 1).

\begin{tabular}{|c|c|c|c|c|c|}
\hline \multirow[t]{2}{*}{ Study } & \multirow[t]{2}{*}{ GEO accession } & \multicolumn{2}{|c|}{ Numbers } & \multirow[t]{2}{*}{ Sample } & \multirow[t]{2}{*}{ Platform } \\
\hline & & AS & Controls & & \\
\hline Study 1 & GSE41038 & 2 & 7 & Synovium & GPL6883, Illumina HumanRef-8 v3.0 expression beadchip \\
\hline Study 2 & GSE39340 & 5 & 7 & Synovium & GPL10558, Illumina HumanHT-12 V4.0 expression beadchip \\
\hline Study 3 & GSE25101 & 16 & 16 & PBMC & GPL6947, Illumina HumanHT-12 V3.0 expression beadchip \\
\hline Study 4 & GSE11886 & 8 & 9 & Macrophage & GPL570, Affymetrix U133 plus 2.0 \\
\hline
\end{tabular}




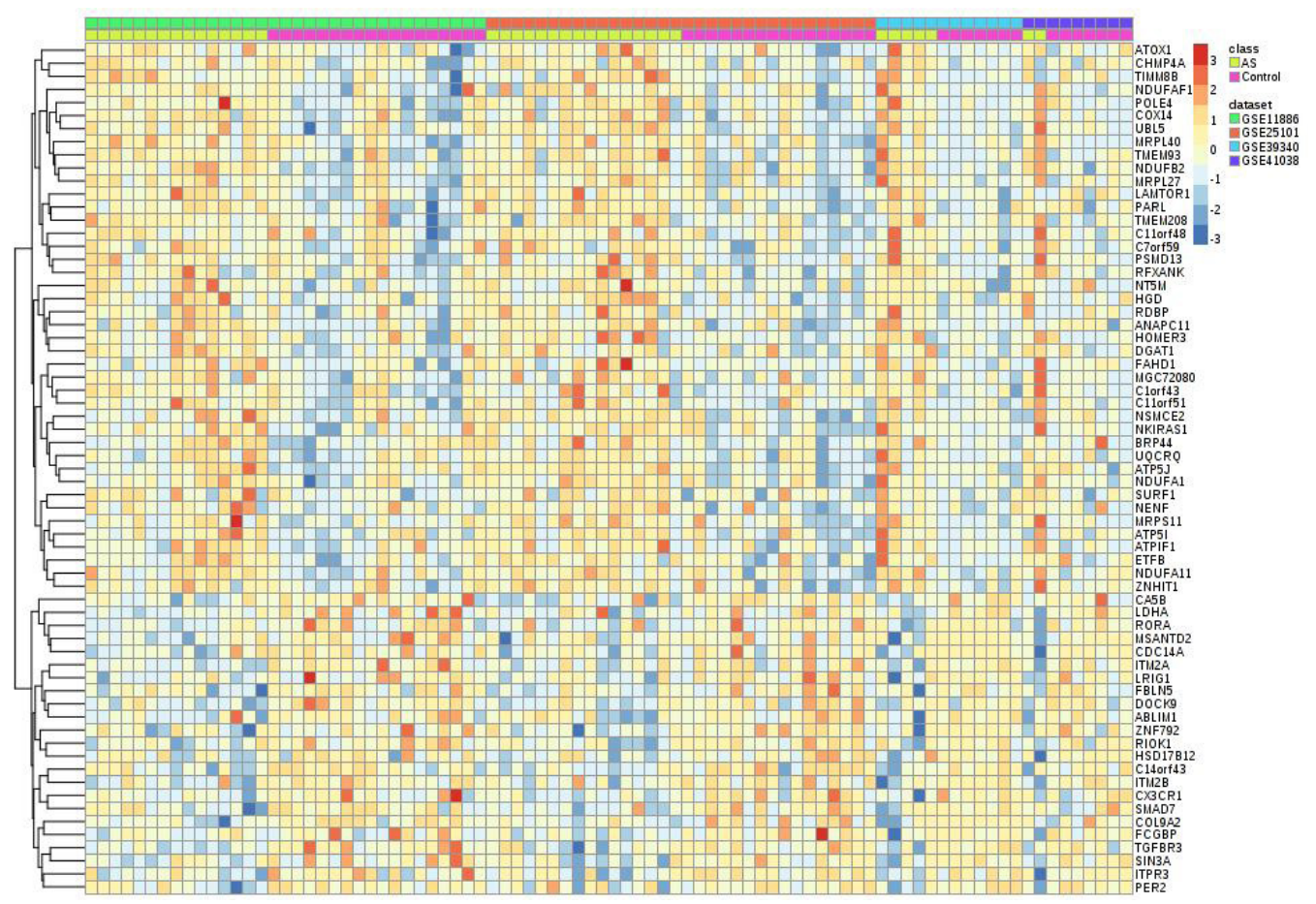

Figure 1. Heat map visualization of the differential expression of particular genes across different datasets (rowwise comparison). The map was generated by re-scaling individual datasets to prevent domination by study-specific effects. AS = ankylosing spondylitis.

\section{Meta-analysis of gene expression in AS}

We used a random-effects model of effect size (ES) measurements to integrate gene expression patterns and incorporate between-study heterogeneities because the estimated Q value was not in a chi-squared distribution. We selected the $\mathrm{DE}$ genes with a $\mathrm{P}$ value $<0.05$. There were 51 "gained" genes and 521 "lost" genes in this meta-analysis (Figure 2). "Gained" genes are the DE genes uniquely identified in the meta-analysis (Xia et al., 2013). These genes showed relatively weak but consistent expression profiles across the individual datasets. Their detection benefits from a larger number of samples and therefore they have more confidence to be declared as DE genes. "Lost" genes were those identified as DE genes in any individual analysis, but not in the meta-analysis. These genes either show conflicting changes in expression profiles between studies, or very large variation across different studies (arising from i.e. batch effect or system bias due to different platforms).

\section{Identification of DE genes in AS}

We identified a total of 65 genes across the four studies that were consistently DE in AS. Among the 65 DE genes, 23 were upregulated and 42 were downregulated. A list of the top 20 most significantly up- or downregulated genes is presented in Tables 2 and 3 . The 
upregulated gene with the largest ES (-1.2628) was integral membrane protein 2A (ITM2A), which is expressed by CD4+ T cells and plays a role in activation of T cells (Kirchner and Bevan, 1999). The upregulated gene with the lowest $P$ value (0.005673) was transforming growth factor beta receptor III (TGFBR3), which, as part of the TGF pathway, is a true bone mineral density-associated gene (Xiong et al., 2009). These genes have not been previously reported to be associated with AS. Many consistently DE genes from the datasets are involved in immune dysregulation (Table 2). The downregulated gene with the largest ES (1.2299) was mitochondrial ribosomal protein S11 (MRPS11), which plays a role in protein synthesis within the mitochondrion (Emdadul Haque et al., 2008). The downregulated gene with the lowest $\mathrm{P}$ value (0.005673) was homogentisate 1,2-dioxygenase $(H G D)$, which is involved in the catabolism of the amino acids tyrosine and phenylalanine (Zatkova et al., 2012). HGD deficiency is associated with bone deformity in AS (Balaban et al., 2006). Further studies are needed to elucidate the function of these DE genes in the pathogenesis of AS (Table 3).

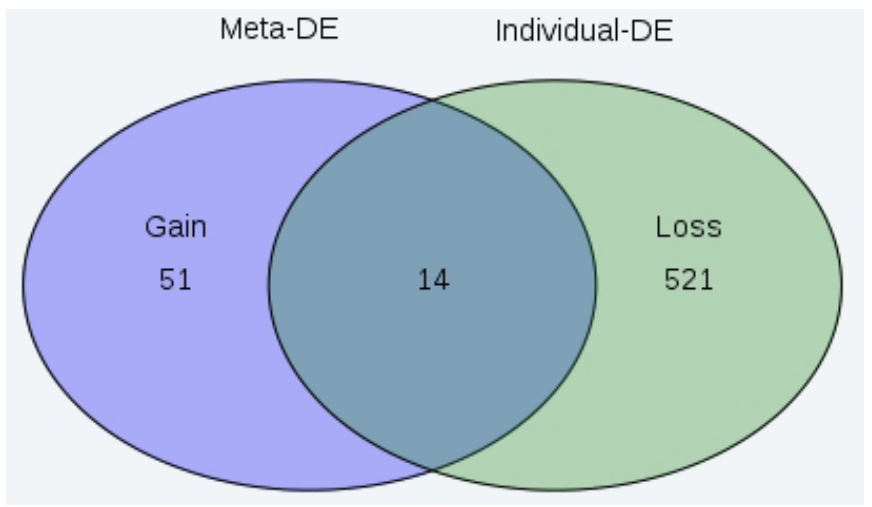

Figure 2. Venn diagram showing overlap between DE genes identified in the meta-analysis (meta-DE) and those from each individual data analysis (individual-DE). DE = differentially expressed.

Table 2. Top 20 upregulated genes in ankylosing spondylitis.

\begin{tabular}{|c|c|c|c|c|}
\hline Entry ID & Gene symbol & Combined ES & P value & Gene name \\
\hline 9452 & ITM2A & -1.2628 & 0.020951 & Integral membrane protein $2 \mathrm{~A}$ \\
\hline 7049 & TGFBR3 & -1.1914 & 0.005673 & Transforming growth factor, beta receptor III \\
\hline 1298 & COL9A2 & -1.0516 & 0.015983 & Collagen, type IX, alpha 2 \\
\hline 1524 & $C X 3 C R 1$ & -0.98613 & 0.021885 & Chemokine (C-X3-Cmotif) receptor 1 \\
\hline 83732 & RIOK1 & -0.98283 & 0.021885 & Rio kinase 1 \\
\hline 23348 & DOCK9 & -0.98149 & 0.021885 & Dedicator of cytokinesis 9 \\
\hline 4092 & SMAD7 & -0.9634 & 0.025091 & Smad family member $7 \underline{1}$ \\
\hline 8864 & PER2 & -0.94092 & 0.042671 & Period circadian clock 2 \\
\hline 25942 & $\operatorname{SIN} 3 A$ & -0.93457 & 0.029029 & Sin3 transcription regulator homolog A \\
\hline 11238 & $C A 5 B$ & -0.9231 & 0.033352 & Carbonic anhydrase $\mathrm{Vb}$, mitochondrial \\
\hline 3710 & ITPR3 & -0.92061 & 0.033352 & Inositol 1,4,5-trisphosphate receptor, type 3 \\
\hline 79684 & MSANTD2 & -0.91939 & 0.033352 & $\mathrm{Myb} /$ sant-like DNA-binding domain containing 2 \\
\hline 91748 & C14orf43 & -0.91889 & 0.033352 & Chromosome 14 open reading frame 43 \\
\hline 3939 & LDHA & -0.90633 & 0.048294 & Lactate dehydrogenase A \\
\hline 9445 & ITM2B & -0.89158 & 0.040075 & Integral membrane protein $2 \mathrm{~b}$ \\
\hline 10516 & FBLN5 & -0.89038 & 0.038737 & Fibulin 5 \\
\hline 26018 & LRIGI & -0.88739 & 0.039607 & Leucine-rich repeats and immunoglobulin-like domains 1 \\
\hline 8556 & $C D C 14 A$ & -0.88513 & 0.040075 & Cell division cycle $14 \mathrm{a}$ \\
\hline 8857 & $F C G B P$ & -0.87546 & 0.0407 & $\mathrm{Fc}$ fragment of IgG binding protein \\
\hline 6095 & RORA & -0.86465 & 0.042671 & Rar-related orphan receptor A \\
\hline
\end{tabular}

$\mathrm{ES}=$ effect size. 
Table 3. Top 20 downregulated genes in ankylosing spondylitis.

\begin{tabular}{lllll}
\hline Entry ID & Gene & Combined ES & P value & Description \\
\hline 64963 & MRPS11 & 1.2299 & 0.040075 & Mitochondrial ribosomal protein S11 \\
3081 & HGD & 1.221 & 0.005673 & Homogentisate 1,2-dioxygenase \\
59286 & UBL5 & 1.2096 & 0.025091 & Ubiquitin-like 5 \\
522 & ATP5J & 1.1596 & 0.006938 & ATP synthase, H+ transporting, mitochondrial Focomplex, subunit F6 \\
8625 & RFXANK & 1.0991 & 0.042375 & Regulatory factor X-associated ankyrin-containing protein \\
4708 & NDUFB2 & 1.0834 & 0.015464 & NADH dehydrogenase (ubiquinone) 1 beta subcomplex, 2, 8 kDa \\
84987 & COX14 & 1.0706 & 0.015464 & Cytochrome C oxidase assembly homolog 14 \\
64976 & MRPL40 & 1.0693 & 0.015464 & Mitochondrial ribosomal protein L40 \\
25906 & C11orf51 & 1.0666 & 0.034987 & Chromosome 11 open reading frame 51 \\
9454 & HOMER3 & 1.0507 & 0.015983 & Homer homolog 3 \\
126328 & NDUFA11 & 1.038 & 0.015983 & NADH dehydrogenase (ubiquinone) 1 alpha subcomplex, 11, 14.7 kDa \\
83460 & TMEM93 & 1.0299 & 0.017366 & Transmembrane protein 93 \\
4694 & NDUFA1 & 1.0262 & 0.048294 & NADH dehydrogenase (ubiquinone) 1 alpha subcomplex, 1, 7.5 kDa \\
55004 & LAMTORI & 1.025 & 0.018842 & Late endosomal/lysosomaladaptor, MAPK and MTOR activator $1{ }^{1}$ \\
5719 & PSMD13 & 1.0056 & 0.020951 & Proteasome (prosome, macropain) 26S subunit, non-ATPase, 13 \\
475 & ATOX1 & 0.99681 & 0.020951 & ATX1 antioxidant protein 1 homolog \\
26521 & TIMM8B & 0.9956 & 0.040075 & Translocaseof inner mitochondrial membrane 8 homolog B \\
286053 & NSMCE2 & 0.98005 & 0.021885 & Non-SMC element 2, MMS21 homolog \\
521 & ATP5I & 0.97762 & 0.021885 & ATP synthase, H+ transporting, mitochondrial Focomplex, subunit E \\
51264 & MRPL27 & 0.97719 & 0.021885 & Mitochondrial ribosomal protein L27 \\
\hline
\end{tabular}

ES $=$ effect size.

\section{Functional analysis}

To identify the biological processes associated with gene expression changes in AS, we performed GO analysis on the DE genes. We identified 210 significant enrichments in the DE genes, which were classified into $10 \mathrm{GO}$ categories (Figure 3 ). The most significant enrichment was in the GO category respiratory electron transport chain $\left(\mathrm{P}=1.67 \times 10^{-9}\right)$. Other significant GO categories included cellular respiration $\left(\mathrm{P}=1.70 \times 10^{-9}\right)$ and the electron transport chain $\left(\mathrm{P}=2.37 \times 10^{-8}\right)$ (Table 4). The identified GO terms further divided into a small number of categories: biological regulation, cellular metabolic process, small molecule metabolic process, cellular component organization, generation of precursor metabolites and energy, energy derivation by oxidation of organic compounds, oxidation-reduction process, cellular respiration, respiratory electron transport chain, electron transport chain, and others (Figure 3). To further investigate the functions of the $65 \mathrm{DE}$ genes, we mapped them to the KEGG database (Table 5). We identified 42 significant pathways based on the KEGG database analysis, the most significant of which was transcriptional misregulation in cancer $(\mathrm{P}=$ $0.0025629)$. Other significant pathways included circadian rhythm - mammal $(\mathrm{P}=0.0034372)$ and fat digestion and absorption $(\mathrm{P}=0.035981$; Table 5).

\section{DISCUSSION}

Many genes are DE in inflammatory diseases such as AS (Pimentel-Santos et al., 2011), and the challenge is to identify the most important genes and pathways associated with a particular disease. These in turn will aid in the development of treatments and therapies for the disorder. Therefore, characterization of the molecular and cellular events that occur during the pathogenesis of AS is an important endeavor. To do this, we chose a meta-analysis approach that combined DE genes from multiple microarray datasets to identify genes that were consistently DE and that reached statistical significance, and performed GO enrichment analysis using hypergeometric tests and pathway analysis using KEGG (Xia et al., 2013). 

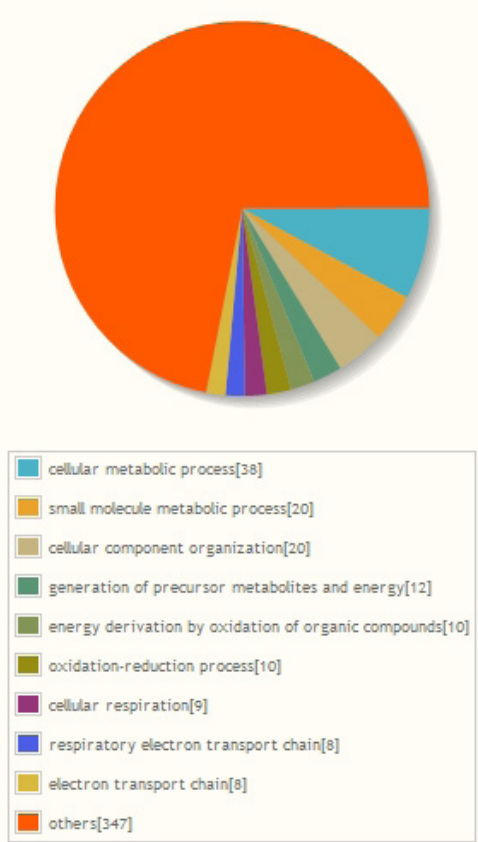

Figure 3. Summary of the enriched GO terms for the list of DE genes from patients with AS as compared to controls. Number of DE genes in each category listed in brackets. $\mathrm{GO}=$ gene ontology; $\mathrm{DE}=$ differentially expressed; AS = ankylosing spondylitis.

Table 4. Top 15 enriched GO terms from the active genes in ankylosing spondylitis.

\begin{tabular}{|c|c|c|c|}
\hline GO ID & Term & $P$ value & Genes \\
\hline GO:0022904 & Respiratory electron transport chain & $1.67 \mathrm{E}-09$ & $\begin{array}{l}\text { ATP } 5 J ; \text { NDUFB } 2 \text {; NDUFA11; ATP } 51 ; \text { UQCRQ; } \\
\text { ETFB; NDUFAF1; NDUFA1 }\end{array}$ \\
\hline GO:0045333 & Cellular respiration & $1.70 \mathrm{E}-09$ & $\begin{array}{l}\text { ATP5J; NDUFB } 2 ; \text { NDUFA11; ATP5I; SURF1; } \\
\text { UQCRQ; ETFB; NDUFAF1; NDUFA1 }\end{array}$ \\
\hline GO:0022900 & Electron transport chain & 2.37E-08 & $\begin{array}{l}\text { ATP } 5 J ; \text { NDUFB } 2 ; \text { NDUFA11; ATP5I; UQCRQ; } \\
\text { ETFB; NDUFAF1; NDUFA1 }\end{array}$ \\
\hline GO:0006091 & Generation of precursor metabolites and energy & $2.76 \mathrm{E}-08$ & $\begin{array}{l}\text { ATP } 5 J ; \text { NDUFB } 2 ; \text { NDUFA11; ATP } 5 I ; \text { SURF } 1 ; \\
\text { ATPIF1; UQCRQ; ITPR } 3 ; \text { ETFB; } \\
\text { NDUFAF1; NDUFA1; LDHA }\end{array}$ \\
\hline GO:0015980 & Energy derivation by oxidation of organic compounds & $1.16 \mathrm{E}-07$ & $\begin{array}{l}\text { ATP5J; NDUFB2; NDUFA11; ATP 5I; SURF1; } \\
\text { UQCRQ; ITPR } 3 ; \text { ETFB; NDUFAF1; NDUFA1 }\end{array}$ \\
\hline GO:0055114 & Oxidation-reduction process & 1.73E-05 & $\begin{array}{l}\text { ATP5J; NDUFB } 2 \text {; NDUFA11; ATP } 51 ; \text { SURF } 1 \text {; } \\
\text { UQCRQ; ITPR } ; \text { ETFB; NDUFAF1; NDUFA1 }\end{array}$ \\
\hline GO:0006119 & Oxidative phosphorylation & $5.48 \mathrm{E}-05$ & NDUFB $2 ;$ SURF1; NDUFAF1; NDUFA1 \\
\hline GO:0044281 & Small molecule metabolic process & $6.91 \mathrm{E}-05$ & $\begin{array}{l}\text { HGD; ATP } 5 J ; \text { NDUFB } 2 ; \text { NDUFA11; PSMD13; } \\
\text { ATP5I; SURF1; ATPIFI; UQCRQ; SIN } 3 A ; \text { CA5B; } \\
\text { ITPR3; ETFB; RORA; HSD17B12; NKIRAS1; } \\
\text { NT5M; NDUFA1; LDHA; DGAT1 }\end{array}$ \\
\hline GO:0006120 & Mitochondrial electron transport, NADH to ubiquinone & 0.00028854 & NDUFB $2 ;$ NDUFAF1; NDUFA1 \\
\hline GO:0008535 & Respiratory chain complex IV assembly & 0.000449877 & COX14; SURF1 \\
\hline GO:0030071 & Regulation of mitotic metaphase/anaphase transition & 0.000489945 & NSMCE2; C11orf51; ANAPC11 \\
\hline GO:0042773 & ATP synthesis coupled electron transport & 0.000637252 & NDUFB2; NDUFAF1; NDUFA1 \\
\hline GO:0042775 & Mitochondrial ATP synthesis coupled electron transport & 0.000637252 & NDUFB2; NDUFAF1; NDUFA1 \\
\hline GO:0006754 & ATP biosynthetic process & 0.000720407 & ATP5J; ATP5I; SURF1 \\
\hline GO:0042776 & Mitochondrial ATP synthesis coupled proton transport & 0.000965788 & ATP5J; ATP5I \\
\hline
\end{tabular}

$\mathrm{GO}=$ gene ontology. 


\begin{tabular}{ll} 
Table 5. List of the top 15 pathways based on KEGG analysis. \\
\hline \\
\hline Pathway & P value \\
\hline Transcriptional misregulation in cancer & 0.0025629 \\
Circadian rhythm - mammal & 0.0034372 \\
Fat digestion and absorption & 0.035981 \\
Pyrimidine metabolism & 0.062147 \\
Glutamatergic synapse & 0.062147 \\
Oocyte meiosis & 0.069928 \\
Cell cycle & 0.088843 \\
Fatty acid elongation & 0.089524 \\
Nicotinate and nicotinamide metabolism & 0.10062 \\
Pancreatic secretion & 0.11523 \\
Tyrosine metabolism & 0.12603 \\
Cysteine and methionine metabolism & 0.1296 \\
Purine metabolism & 0.14024 \\
Pyruvate metabolism & 0.15423 \\
Taste transduction & 0.16801 \\
\hline
\end{tabular}

KEGG $=$ Kyoto encyclopedia of genes and genomes.

We performed a meta-analysis using four publicly available GEO datasets to identify common biological mechanisms involved in the pathogenesis of AS. We identified genes that were consistently over- or under-expressed, significant GO enrichments, and pathways associated with AS. In total, 65 genes across the four studies were consistently DE in AS (23 up- and 42 downregulated). The upregulated gene with the lowest $\mathrm{P}$ value (0.005673) was TGFBR3 and the upregulated gene with the largest ES $(-1.2628)$ was ITM2A. Although the roles and association of these genes with AS have not yet been reported, ITM $2 A$ is involved in osteoand chondrogenic cellular differentiation (cells responsible for the development of bone and cartilage, respectively) (Deleersnijder et al., 1996). ITM2A is also involved in the activation of $\mathrm{T}$ cells in the immune system (Kirchner and Bevan, 1999) and in myocyte differentiation (Van den Plas and Merregaert, 2004). Furthermore, some of the upregulated genes, e.g., ITM2A and collagen, type IX, alpha 2 (COL9A2), might modulate cartilage and bone metabolism leading to AS progression. Our meta-analysis of gene expression data also revealed upregulation of chemokine $\mathrm{C}-\mathrm{X} 3-\mathrm{C}$ motif receptor $1(C X 3 C R 1)$, consistent with previous microarray results of proinflammatory profiles in AS (Pimentel-Santos et al., 2011). Additional specific upregulated genes are involved in mediation of inflammation and in the chemotaxis pathway. The downregulated gene with the lowest $\mathrm{P}$ value $(0.005673)$ was $H G D$, which is involved in the catabolism of the amino acids tyrosine and phenylalanine. HGD deficiency is relevant to the bone deformity of AS, as patients with HGD deficiency experience progressive kyphosis, obliteration of intervertebral spaces, and marginal intervertebral osteophytes resembling the syndesmophytes in AS (Zatkova et al., 2012).

The most significant GO enrichment amongst the list of 210 categories was the respiratory electron transport chain $\left(\mathrm{P}=1.67 \times 10^{-9}\right)$. Other significant $\mathrm{GO}$ categories included cellular respiration $\left(\mathrm{P}=1.70 \times 10^{-9}\right)$ and the electron transport chain $\left(\mathrm{P}=2.37 \times 10^{-8}\right)$. Complexes I and II of the electron transport chain release reactive oxygen species (ROS) exclusively in the mitochondrial matrix, whereas complex III generates ROS on both sides of the mitochondrial inner membrane (Sena and Chandel, 2012). ROS can damage DNA, RNA, and proteins, and play a key role in the pathogenesis of inflammatory diseases like AS (Özenirler et al., 2013). Amongst the 42 pathways in our KEGG analysis, transcriptional misregulation in cancer, circadian rhythm, and fat digestion and absorption were the most differently regulated in 
AS. Transcriptional misregulation is known to contribute to tumorigenesis, and overexpressed oncogenic transcription factors alter the autoregulatory circuitry of the cell (Lee and Young, 2013). Thus, transcriptional misregulation can contribute to cancer, autoimmunity, and inflammation (Lee and Young, 2013). The GO categories and KEGG pathways identified in this study merit further study and validation.

The present study has some limitations that require consideration. First, heterogeneity and confounding factors may have distorted the analysis. Clinical samples might have been heterogeneous with respect to clinical activity, severity, or gender. Second, the inflammatory nature of AS results in changes in gene expression in the white blood cell population and in the synovium. There are differences in gene expression between the synovium and blood that were not considered. However, our meta-analysis integrated samples from different tissues, which might have enabled us to detect genes that we would otherwise have missed in subgroup analysis. Third, the numbers of studies and samples included in this meta-analysis were small. Small sample size and study number may not result in enough power to detect true gene expression changes involved in the pathogenesis of AS. Fourth, the magnitudes of the changes in gene expression identified were not large.

In conclusion, meta-analysis of gene expression profiling provided a global overview of differential gene expression in AS identifying 65 DE genes (23 up- and 42 downregulated genes). Integrated pathway analysis of the differentially regulated genes indicated roles in electron transport, inflammation, and various other processes. Our meta-analysis revealed previously unknown transcriptional changes in AS. Identification of the gene expression changes observed in AS will provide valuable insights into the pathogenesis of AS. Further functional studies might provide additional insights into the role of the differentially regulated genes in the pathophysiology of AS.

\section{ACKNOWLEDGMENTS}

Research received no specific grants from any funding agency in the public, commercial, or not-for-profit sectors.

\section{REFERENCES}

Balaban B, Taskaynatan M, Yasar E, Tan K, et al. (2006). Ochronotic spondyloarthropathy: spinal involvement resembling ankylosing spondylitis. Clin. Rheumatol. 25: 598-601.

Barrett T, Troup DB, Wilhite SE, Ledoux P, et al. (2011). NCBI GEO: archive for functional genomics data sets - 10 years on. Nucleic Acids Res. 39: D1005-D1010.

Bolstad BM, Irizarry RA, Astrand M and Speed TP (2003). A comparison of normalization methods for high density oligonucleotide array data based on variance and bias. Bioinformatics 19: 185-193.

Brown MA, Wordsworth BP and Reveille JD (2002). Genetics of ankylosing spondylitis. Clin. Exp. Rheumatol. 20: S43-49.

Choi JK, Yu U, Kim S and Yoo OJ (2003). Combining multiple microarray studies and modeling interstudy variation. Bioinformatics 19: i84-i90.

Choi SJ, Rho YH, Ji JD, Song GG, et al. (2006). Genome scan meta-analysis of rheumatoid arthritis. Rheumatology 45: 166-170.

Deleersnijder W, Hong G, Cortvrindt R, Poirier C, et al. (1996). Isolation of markers for chondro-osteogenic differentiation using cDNA library subtraction. Molecular cloning and characterization of a gene belonging to a novel multigene family of integral membrane proteins. J. Biol. Chem. 271: 19475-19482.

DerSimonian R and Laird N (1986). Meta-analysis in clinical trials. Control Clin. Trials 7: 177-188.

Emdadul Haque M, Grasso D, Miller C, Spremulli LL, et al. (2008). The effect of mutated mitochondrial ribosomal proteins S16 and S22 on the assembly of the small and large ribosomal subunits in human mitochondria. Mitochondrion 8: 254-261. 
Falcon S and Gentleman R (2007). Using GO stats to test gene lists for GO term association. Bioinformatics 23: 257-258. Golub TR, Slonim DK, Tamayo P, Huard C, et al. (1999). Molecular classification of cancer: class discovery and class prediction by gene expression monitoring. Science 286: 531-537.

Griffith OL, Melck A, Jones SJ and Wiseman SM (2006). Meta-analysis and meta-review of thyroid cancer gene expression profiling studies identifies important diagnostic biomarkers. J. Clin. Oncol. 24: 5043-5051.

Hua L, Li DG, Lin H, Li L, et al. (2010). The correlation of gene expression and co-regulated gene patterns in characteristic KEGG pathways. J. Theor. Biol. 266: 242-249.

Kirchner J and Bevan MJ (1999). ITM2A is induced during thymocyte selection and T cell activation and causes downregulation of CD8 when overexpressed in CD4(+)CD8(+) double positive thymocytes. J. Exp. Med. 190: 217-228.

Lee TI and Young RA (2013). Transcriptional regulation and its misregulation in disease. Cell 152: 1237-1251.

Lee YH and Nath SK (2005). Systemic lupus erythematosus susceptibility loci defined by genome scan meta-analysis. Hum. Genet. 118: 434-443.

Lee YH, Rho YH, Choi SJ, Ji JD, et al. (2005a). Ankylosing spondylitis susceptibility loci defined by genome-search meta-analysis. J. Hum. Genet. 50: 453-459.

Lee YH, Witte T, Momot T, Schmidt RE, et al. (2005b). The mannose-binding lectin gene polymorphisms and systemic lupus erythematosus: two case-control studies and a meta-analysis. Arthritis Rheum. 52: 3966-3974.

Özenirler S, Degertekin CK, Erkan G, Elbeğ S, et al. (2013). Serum liver fatty acid binding protein shows good correlation with liver histology in NASH. Hepatogastroenterology 60: 1095-1100.

Pimentel-Santos FM, Ligeiro D, Matos M, Mourão AF, et al. (2011). Whole blood transcriptional profiling in ankylosing spondylitis identifies novel candidate genes that might contribute to the inflammatory and tissue-destructive disease aspects. Arthritis Res. Ther. 13: R57.

Ramasamy A, Mondry A, Holmes CC and Altman DG (2008). Key issues in conducting a meta-analysis of gene expression microarray datasets. PLoS Med. 5: e184.

Rung J and Brazma A (2013). Reuse of public genome-wide gene expression data. Nat. Rev. Genet. 14: 89-99.

Sena LA and Chandel NS (2012). Physiological roles of mitochondrial reactive oxygen species. Mol. Cell 48: 158-167.

Siddiqui AS, Delaney AD, Schnerch A, Griffith OL, et al. (2006). Sequence biases in large scale gene expression profiling data. Nucleic Acids Res. 34: e83.

Van den Plas D and Merregaert J (2004). Constitutive overexpression of the integral membrane protein Itm2A enhances myogenic differentiation of C2C12 cells. Cell Biol. Int. 28: 199-207.

van der Linden S, Valkenburg HA and Cats A (1984). Evaluation of diagnostic criteria for ankylosing spondylitis. A proposal for modification of the New York criteria. Arthritis Rheum. 27: 361-368.

Xia J, Fjell CD, Mayer ML, Pena OM, et al. (2013). INMEX - a web-based tool for integrative meta-analysis of expression data. Nucleic Acids Res. 41: W63-70.

Xiong DH, Liu XG, Guo YF, Tan LJ, et al. (2009). Genome-wide association and follow-up replication studies identified ADAMTS18 and TGFBR3 as bone mass candidate genes in different ethnic groups. Am. J. Hum. Genet. 84: 388-398.

Zatkova A, Sedlackova T, Radvansky J, Polakova H, et al. (2012). Identification of 11 novel homogentisate 1,2 dioxygenase variants in alkaptonuria patients and establishment of a novel LOVD-based HGD mutation database. JIMD Rep. 4: $55-65$. 